Les Cahiers du Gres

\title{
L'éveil aux langues : des enfants du primaire parlent des langues et de la diversité linguistique
}

\section{Érica Maraillet et Françoise Armand}

Volume 6, numéro 2, hiver 2006

URI : https://id.erudit.org/iderudit/014445ar

DOI : https://doi.org/10.7202/014445ar

Aller au sommaire du numéro

Éditeur(s)

Groupe de Recherche Ethnicité et Société

CEETUM

ISSN

1499-0431 (imprimé)

1499-044X (numérique)

Découvrir la revue

Citer cet article

Maraillet, É. \& Armand, F. (2006). L'éveil aux langues : des enfants du primaire parlent des langues et de la diversité linguistique. Les Cahiers du Gres, 6(2),

17-34. https://doi.org/10.7202/014445ar
Résumé de l'article

À l'heure de la mondialisation et dans le cadre du renouvellement des réflexions sur l'avenir linguistique du Québec, le thème de la diversité linguistique est devenu depuis peu un sujet d'actualité, comme en témoigne le récent ouvrage Le français au Québec, les nouveaux défis de Stefanescu et Georgeault (2005). En effet, cet ouvrage fait émerger le besoin d'arrimer la promotion et la protection de la langue française, tant sur la scène mondiale qu'au Québec, à la reconnaissance et la valorisation de la diversité linguistique. Alors que les langues et la diversité linguistique sont l'objet de nombreux questionnements à tous les niveaux de la sphère publique, à Montréal comme ailleurs, cet article propose d'apporter un éclairage sur ce que des enfants de $5^{\mathrm{e}}$ et $6^{\mathrm{e}}$ années du primaire, issus de l'immigration, perçoivent de ces débats. Afin de susciter chez eux une réflexion plus poussée, cette recherche a été réalisée dans le cadre de l'implantation d'un projet d'éveil aux langues (ÉLODiL), dont les activités ont servi de déclencheur et ont permis d'établir un rapport de confiance entre les élèves et les chercheurs. 


\section{DES ENFANTS DU PRIMAIRE PARLENT DES LANGUES \\ ET DE LA DIVERSITÉ LINGUISTIQUE}

\section{Érica Maraillet Françoise Armand}

\section{Résumé / Abstract}

À l'heure de la mondialisation et dans le cadre du renouvellement des réflexions sur l'avenir linguistique du Québec, le thème de la diversité linguistique est devenu depuis peu un sujet d'actualité, comme en témoigne le récent ouvrage Le français au Québec, les nouveaux défis de Stefanescu et Georgeault (2005). En effet, cet ouvrage fait émerger le besoin d'arrimer la promotion et la protection de la langue française, tant sur la scène mondiale qu'au Québec, à la reconnaissance et la valorisation de la diversité linguistique. Alors que les langues et la diversité linguistique sont l'objet de nombreux questionnements à tous les niveaux de la sphère publique, à Montréal comme ailleurs, cet article propose d'apporter un éclairage sur ce que des enfants de $5^{\mathrm{e}}$ et $6^{\mathrm{e}}$ années du primaire, issus de l'immigration, perçoivent de ces débats. Afin de susciter chez eux une réflexion plus poussée, cette recherche a été réalisée dans le cadre de l'implantation d'un projet d'éveil aux langues (ÉLODiL), dont les activités ont servi de déclencheur et ont permis d'établir un rapport de confiance entre les élèves et les chercheurs.

In the midst of globalisation, and in the context of certain new ideas about the linguistic future of Quebec, Stefanescu and Georgeault's recent book, Le français au Québec, les nouveaux défis, notes that linguistic diversity has recently attracted wide attention. Indeed, this book reveals the need to articulate the promotion and protection of the French language, with the recognition and valorisation of linguistic diversity on the international scene as well as in Quebec. In Montreal as elsewhere in Quebec, languages and linguistic diversity are being examined at all levels of the public sphere. This article attempts to shed some light on what children of immigrant origin, in the fifth and sixth grades of a multiethnic primary school, perceive regarding the debates around this topic. This study took place in the context of a language awareness project (ÉLODiL) oriented to fostering a thought provoking and trusting environment for pupils and researchers.

Mots clés : attitudes, représentations, diversité linguistique, élèves du primaire, éveil aux langues, Montréal.

Keywords: attitudes, perceptions, linguistic diversity, students in primary school, language awareness, Montreal. 
À l'heure de la mondialisation, et dans le cadre du renouvellement des réflexions sur l'avenir linguistique du Québec, le thème de la diversité linguistique est devenu depuis peu un sujet d'actualité, comme en témoigne le tout récent ouvrage Le français au Québec, les nouveaux défis de Stefanescu et Georgeault (2005). En effet, cet ouvrage souligne le besoin d'arrimer la promotion et la protection de la langue française, tant sur la scène mondiale qu'au Québec, à la reconnaissance et la valorisation de la diversité linguistique. À Montréal comme ailleurs, alors que les langues et la diversité linguistique sont l'objet de questionnements à tous les niveaux de la sphère publique, cet article propose d'apporter un éclairage sur ce que des enfants de $5^{\mathrm{e}}$ et $6^{\mathrm{e}}$ années du primaire, issus de l'immigration, perçoivent de ces débats. Afin de susciter chez eux une réflexion plus poussée, cette recherche ${ }^{1}$ a été réalisée dans le cadre de l'implantation d'un projet d'éveil aux langues (ÉLODiL), dont les activités ont servi de déclencheur et ont permis d'établir un rapport de confiance entre les élèves et les chercheurs. Avant d'expliciter la méthode et les résultats de cette recherche, nous commencerons par décrire le contexte linguistique montréalais. Ensuite, nous présenterons l'importance accordée à la diversité linguistique dans les différents programmes du ministère de l'Éducation, du Loisir et du Sport du Québec ainsi que les études qui ont porté sur les représentations et les attitudes de différents acteurs à l'égard des langues.
ConteXTe LINGUISTIQUE MONTRÉALAIS

L'histoire du Québec est marquée par un conflit linguistique entre les anglophones et les francophones qui y vivent, conflit qui s'est concrétisé, à partir des années 1960, par ce qui sera plus tard appelé la Révolution tranquille. La division de ces deux communautés linguistiques s'est traduite en une division des commissions scolaires : l'une, protestante, pour les anglophones et l'autre, catholique, pour les francophones ${ }^{2}$. Au fur et à mesure des différentes vagues d'immigration vers le Québec, plusieurs groupes ethniques ont intégré l'une ou l'autre des deux commissions et ont quelque peu complexifié leur structure : des soussystèmes franco-protestant et anglocatholique ont ainsi été créés. Ces soussystèmes ont fait en sorte que l'intégration des immigrants dans les écoles québécoises n'a jamais vraiment été envisagée comme un enjeu majeur de société, jusqu'à ce que, dans le contexte de la Révolution tranquille, qui a redéfini l'identité collective du Québec, on s'aperçoive que la majorité des immigrants fréquentaient le secteur anglo-protestant (McAndrew et Proulx 2000). En effet, le groupe francophone au Québec (les Canadiens français) était considéré comme une minorité ethnique au sein du Canada, avant qu'il ne se transforme en un groupe majoritaire dans la province du Québec (les Québécois) et en une communauté d'accueil pour les immigrants (McAndrew et Lamarre 1996). Tout au long de ce processus, une 
série de lois ont été promulguées, dont la plus importante est la Loi 101, la Charte de la langue française (1977), qui prescrit l'utilisation de la langue française dans les entreprises et les établissements, en politique et plus généralement dans la vie publique québécoise. Bien que reconnues comme nécessaires à la survie de la langue française par de nombreux représentants des différentes communautés linguistiques (les anglophones, les francophones et les locuteurs allophones de diverses origines ${ }^{3}$ ), ces lois ont aussi été largement contestées par d'autres représentants de ces mêmes communautés, et ce au nom de la liberté des droits individuels.

Dans le domaine de l'éducation, l'une des dispositions de la Loi 101 avait pour objectif spécifique d'inverser la tendance des immigrants à placer leurs enfants dans les écoles de langue anglaise, afin de favoriser l'utilisation généralisée de la langue française par la population au Québec. En effet, la Loi 101 dirige, à quelques exceptions près, tous les enfants immigrants nouvellement arrivés dans les écoles francophones. L'impact de cette loi sur les écoles françaises est très important si l'on considère que l'île de Montréal et sa région métropolitaine accueillent la majorité des immigrants reçus au Québec (MRCI 2003). Ainsi, à Montréal, en 2002, plus d'un tiers $(36,3 \%)$ des élèves du primaire et du secondaire des écoles publiques sont scolarisés dans une langue autre que leur langue maternelle; ce pourcentage grimpant à 43,51\% dans la plus grande commission scolaire de Montréal (Comité de gestion de la taxe scolaire de l'île de Montréal 2003), et on recense plus de 150 langues maternelles différentes. Par conséquent, pour les écoles montréalaises de langue française, l'intégration relativement récente d'un très grand nombre d'enfants issus de communautés linguistiques diverses présente un double défi : d'une part, celui de l'enseignement de la langue française, qui vise à assurer l'intégration de sa clientèle allophone à une société francophone (et celui de son usage, qui symbolise le renouvellement de la collectivité francophone) et, d'autre part, la prise en compte de la diversité culturelle et linguistique de ces élèves d'origine immigrante.

\section{CONTEXTE SCOlaIRE ET DIVERSITÉ LINGUISTIQUE}

Que ce soit dans le cadre du Programme de formation de l'école québécoise (MEQ 2001) ou de la Politique d'intégration et d'éducation interculturelle (MEQ 1998), le ministère de l'Éducation, du Loisir et du Sport du Québec (MELS), incite les enseignants québécois, qu'ils œuvrent dans un milieu pluriethnique ou non, à prendre en compte la diversité ethnoculturelle, linguistique et religieuse de la société québécoise dans leurs pratiques d'enseignement. Dans cet article, nous nous intéressons plus particulièrement à la diversité linguistique, et plus précisément aux représentations d'élèves de la fin du primaire sur ce thème. À cet égard, le Ministère promeut l'usage de 
la langue française comme langue commune, mais reconnaît aussi l'importance pour tous les élèves d'apprendre plusieurs langues et de donner une place aux langues d'origine des élèves allophones (MEQ 2001, 1998). Cependant, la promotion de la diversité linguistique ne constitue une priorité ni dans la Politique, ni dans le Programme.

Depuis 1977, on peut aussi noter l'existence des programmes d'enseignement des langues d'origine (PELO) qui visent exclusivement le maintien des langues d'origine des élèves, au moyen de périodes d'enseignement ( 2 heures et demie par semaine), généralement en dehors des heures scolaires, mais dans les locaux de l'école 4 . Deux études majeures ont fait le point sur les PELO : le rapport Chancy (1985), qui propose une évaluation de ces programmes un peu moins d'une décennie après leur implantation, et la recherche amorcée en 1999 par Laurier et Témisjian, dont l'objectif est d'analyser les perceptions de différents acteurs dans la mise en ouvre des PELO au Québec. Le succès et l'expansion de ces programmes y sont considérés comme mitigés. Par ailleurs, le rapport Chancy souligne que « la question de fond touchant ces programmes a trait au type de rapport qu'une société veut établir entre les langues minoritaires et la langue dominante, en l'occurrence le français" et que "la question se complique chez nous compte tenu du rapport entre les communautés française et anglaise»(Chancy $1985: 84$ ). De façon globale, les deux études soulèvent trois problèmes majeurs : 1) le besoin d'un nombre suffisant d'élèves du même âge et d'un même groupe linguistique pour que le programme ait lieu, 2) l'isolement et la complexité de l'arrangement horaire des classes et 3) l'ambiguïté du rôle des différents intervenants (parents, communautés, écoles) dans les démarches d'implantation.

En ce qui a trait aux orientations de l'école et des intervenants au sujet de la langue française en rapport avec l'utilisation des autres langues, l'étude de Beauchesne et Hensler (1987) ${ }^{5}$ montre que c'est surtout dans les écoles primaires que l'on constate la présence de normes explicites et d'une politique linguistique. L'utilisation exclusive du français y est imposée dans une classe sur deux. Les raisons qui justifient cette norme sont la valorisation du développement du français et l'affirmation du statut linguistique de l'école. En ce qui concerne les langues maternelles des élèves, les intervenants pensent en majorité (neuf sur dix) que les élèves en désirent le maintien. Notons que, toujours d'après cette étude, datant de 1987, les répondants sont partagés par rapport aux enjeux de ce désir. La moitié d'entre eux estime que la volonté des élèves de conserver leur langue d'origine a un impact négatif sur le développement du français. L'autre moitié pense que ce désir est plutôt positif et mentionne notamment la valorisation personnelle et le développement d'habiletés linguistiques que cet apprentissage procure. Vingt ans sont passés depuis la 
publication de cette étude, et l'on peut se demander si les perceptions de ces enseignants ont changé, mais aussi, et surtout, quelles sont les représentations des élèves allophones sur ces questions.

Tout récemment, un projet de recherche sur les représentations et les attitudes d'élèves de la fin du primaire à l'égard des langues a été mis en œuvre dans une école pluriethnique à Montréal, dans le cadre d'un projet d'implantation de l'approche Éveil aux langues à Montréal et à Vancouver (Armand et Dagenais ${ }^{6}$ : le projet ÉLODiL- Éveil au Langage et Ouverture à la Diversité Linguistique). C'est au sein de ce projet que la présente étude a été réalisée et que les données qui seront présentées dans cet article ont été recueillies. L'éveil aux langues est une approche pédagogique apparue en Grande-Bretagne dans les années 1980 (Hawkins 1987; Garrett et James 1992). L'objectif principal de cette approche est de permettre aux élèves d'explorer systématiquement la diversité linguistique afin de les préparer à vivre dans des sociétés linguistiquement et culturellement diverses (Armand et Dagenais 2005). Il s'agit, par la manipulation de corpus de différentes langues, de sensibiliser les apprenants : 1) à la diversité des langues et, à travers l'objet langue, de leur faire prendre conscience de la diversité des êtres qui les parlent, et 2) aux différents fonctionnements des langues (aux différentes façons d'exprimer la négation, le genre, le pluriel, etc.).
Dernièrement, Michel Candelier, instigateur d'un projet d'éveil aux langues paneuropéen (Evlang), a publié les résultats de son étude qui visait l'évaluation du programme Evlang (Candelier 2003). Dans cet ouvrage, Candelier souligne que pour constater les effets d'un programme d'éveil aux langues, notamment sur les attitudes d'ouverture à la diversité linguistique des élèves, il faut prévoir un programme d'une durée minimale de 30 heures et qui s'échelonne sur un minimum d'une année scolaire (35 semaines). Au moment de l'implantation du projet ÉLODiL, au sein duquel nous avons recueilli les données pour le présent article, le programme contenait suffisamment d'activités pour couvrir 18 heures. Par ailleurs, les enseignants avaient donné leur accord pour participer au projet entre février et mai (16 semaines). À la lumière des résultats de l'étude de Candelier (2003), l'objectif du projet de recherche plus vaste, dans lequel s'insère cette étude, consistait donc à observer les conditions d'implantation du projet ÉLODiL dans plusieurs classes d'un milieu pluriethnique de Montréal, ainsi qu'à recueillir des données préliminaires sur les représentations et les attitudes d'élèves scolarisés dans des classes régulières et dans des classes d'accueil à l'égard des langues. Il a été prévu de réaliser l'évaluation plus systématique du programme ÉLODiL lorsque celuici aura été implanté sur une plus longue période?. 
Les recherches antérieures portant sur les représentations à l'égard des langues à Montréal ont été effectuées pour examiner les usages et les attitudes linguistiques des élèves dans les écoles primaires et secondaires (Beauchesne et Hensler 1987; McAndrew et al. 1999) ou secondaires seulement (Sénéchal 1987; Locher 1993, 1994). Ces études, réalisées dans la foulée de la vaste entreprise d'aménagement linguistique concrétisée par l'adoption de la Charte de la langue française (Loi 101) de 1977, avaient avant tout le mandat de faire le point sur la place du français et de l'anglais dans les écoles multiethniques montréalaises. L'étude la plus récente à cet égard est celle de McAndrew et al. (1999) qui s'étaient donné l'objectif global d'observer, de façon structurée et en fonction de différents facteurs ${ }^{8}$, les échanges linguistiques informels entre les élèves, afin de tracer un portrait de leur usage du français, de l'anglais ou d'autres langues. Cette étude révèle que le taux de présence de la langue française dans les dix écoles primaires observées se situe entre $67,5 \%$ et $\mathbf{9 9 , 7} \%$, qu'il dépasse $90 \%$ dans six écoles et que l'anglais n'est présent que dans quatre de ces écoles. Par ailleurs, les auteurs soulignent que les langues d'origine sont présentes dans la plupart des écoles, mais à un taux de présence souvent très faible.

Toutefois, à notre connaissance, aucune recherche n'a spécifiquement ciblé les représentations d'élèves du primaire sur la diversité linguistique.
Dans la présente étude, nous nous sommes donc intéressées, au sein du contexte linguistique montréalais, aux représentations et aux attitudes d'élèves du $3^{\text {e }}$ cycle du primaire ${ }^{9}$ à l'égard de la diversité linguistique, dans le cadre d'un projet scolaire d'éveil aux langues.

\section{DIVERSITÉ LINGUISTIQUE ET REPRÉSENTATION SUR LES LANGUES}

Depuis plusieurs années, la diversité linguistique est devenue l'objet de réflexions, notamment sur le plan théorique. La défense des langues dites menacées est assez répandue. Claude Hagège (2002) fait partie des linguistes qui défendent cette cause, soulignant que 25 langues meurent chaque année et, qu'avec elles, disparaissent les cultures humaines dont elles sont le reflet. Le fil conducteur de ce courant semble être le désir de contrecarrer l'omniprésence d'une seule langue, dominante, qui se trouve être actuellement la langue anglaise. Cette position défensive se retrouve de part et d'autre de l'Atlantique, en France comme au Québec, en réponse à l'utilisation grandissante de cette langue. Louis-Jean Calvet (2002) se démarque fortement de ce mouvement. Il souligne l'existence de cette rivalité entre les langues en décrivant l'analogie d'un marché économique des langues, dans lequel celles-ci sont soit valorisées, soit dépréciées. Par ailleurs, il propose un nouveau cadre théorique de la diversité linguistique et de sa place dans l'évolution des dynamiques linguistiques dans le monde. En effet, l'inégalité 
fondamentale affirmée par Calvet est basée sur les différentes fonctions des langues qui répondent à divers besoins linguistiques de leurs locuteurs, à savoir une fonction identitaire, une fonction nationale et une fonction véhiculaire internationale. Ces fonctions peuvent être représentées dans trois langues différentes, deux ou bien une seule (Calvet 2002). Dans cette optique, Calvet propose ce qu'il appelle un modèle gravitationnel dans lequel chaque citoyen devrait pouvoir pratiquer au moins les trois types de langues (1- une langue internationale, globale, 2- une langue normée et standardisée utilisée dans la vie publique de l'État, 3- une langue grégaire ou régionale, écrite ou non, avec un statut ou non). Finalement, Calvet se prononce sur le concept de langue " universelle " qu'il considère plutôt comme une langue complémentaire aux autres langues déjà présentes (2002: 176). Déplorant que la domination internationale de l'anglais soit vue comme une menace à la diversité linguistique, mettant en péril toutes les autres langues, il mentionne que les intérêts pour la promotion de la diversité linguistique peuvent être différents. Il est notamment difficile de voir comment peut s'articuler à la fois la promotion de la langue française et celle de langues beaucoup moins répandues. À la lumière de ce débat, nous serons en mesure de situer les représentations de nos jeunes répondants à l'égard de la diversité linguistique.

À la question « pourquoi étudier les représentations sociales ?", Serge
Moscovici, qui a remis à l'ordre du jour le concept de " représentation sociale " dans le domaine de la psychologie sociale, répond que c'est " pour explorer le côté subjectif de ce qui se passe dans la réalité objective » (1984:12). Bien qu'issues de la psychologie sociale, les notions de représentation et d'attitude sont progressivement devenues des éléments clés pour la compréhension de divers phénomènes dans plusieurs domaines, dont celui de l'éducation. La plus ancienne, la notion d'attitude, désigne communément " un état mental et neuropsychologique de préparation de l'action, organisé à la suite de l'expérience et qui concerne une influence dynamique sur le comportement de l'individu vis-à-vis de tous les objets et de toutes les situations qui s'y rapportent "(Allport $1935: 810$ ). Bien qu'elle soit utilisée fréquemment par les psychologues sociaux, cette définition est loin de faire un consensus. De façon générale, comme le mentionne Candelier, les attitudes sont souvent expliquées par différentes paraphrases, telles que "prédisposition à ", " orientation pour ", et celles-ci tendent à suggérer un lien avec l'action (Candelier 2003 : 139). Quant aux représentations sociales, selon Denise Jodelet, l'idée principale que l'on retrouve est « une manière d'interpréter et de penser notre réalité quotidienne, une forme de connaissance sociale. Et corrélativement, l'activité mentale déployée par les individus et les groupes pour fixer leur position par rapport à des situations, événements, objets et communications qui les concernent » 
L'étude des représentations sociales et des attitudes dans le domaine de l'éducation peut porter sur celles des enseignants tout comme sur celles des élèves. Dans la présente étude, nous avons fait le choix de nous intéresser à celles des élèves, bien que des recherches sur les représentations et les attitudes des enseignants soient tout aussi essentielles. Dans le domaine de l'éducation, un aperçu des différentes études sur les attitudes et les représentations révèle un intérêt évident pour des sujets à partir de l'âge de douze ans environ et pour la population étudiante pré-universitaire et universitaire. Selon Singy (1997), c'est à peu près à l'âge de douze ans que les enfants semblent devenir de plus en plus conscients du jeu des normes qui régulent les pratiques linguistiques utilisées dans la société. Il affirme que les attitudes sont intégrées et développées à partir de cet âge et sont affirmées vers l'âge de $\mathbf{1 8}$ ans. L'étude des représentations et des attitudes envers les langues d'élèves de $5^{\mathrm{e}}$ et de $6^{\mathrm{e}}$ années est donc tout à fait pertinente, permettant de les identifier au début du processus de construction.

Comme nous l'avons déjà noté, qu'il s'agisse de l'apprentissage du français, de l'anglais, des langues d'origine ou d'autres langues, l'enseignement des langues est une priorité dans les programmes québécois. Si l'on s'intéresse aux facteurs sociaux et affectifs qui motivent l'apprentissage des langues secondes, des recherches comme celles de Gardner (1985) montrent que les représentations et les attitudes à l'égard des langues et de leur apprentissage jouent un rôle très important. Il distingue deux volets, les plus étudiés selon lui, qui sont : les attitudes à l'égard de la langue ellemême (appelé volet éducatif) et les attitudes à l'égard de la communauté locutrice de la langue (appelé volet social). Définissant la notion d'attitude comme étant « une réaction à un objet, évaluée par un individu et qui est basée sur ses croyances ou opinions" (traduction ad hoc, Gardner 1985 : 9), Gardner démontre que, bien que les deux volets (et bien d'autres dimensions d'ailleurs) aient un effet sur la motivation à apprendre une langue seconde, ce sont les attitudes et donc les croyances (que nous préférons appeler ici représentations) à l'égard de la langue et de son apprentissage qui ont tendance à être parmi les meilleurs indices de réussite (Gardner 1985; voir aussi Masgoret et Gardner 2003). Dans le cadre de cette recherche, notre objectif a donc été de décrire les représentations et les attitudes à l'égard de différentes langues de quelques élèves d'une école pluriethnique de Montréal.

\section{MÉTHODE}

Dans la présente étude, nous avons examiné les représentations et les attitudes de neuf élèves d'une classe régulière de $5^{\mathrm{e}}$ et $6^{\mathrm{e}}$ années (qualifiée de " classe jumelée ») à l'égard des langues et de la diversité linguistique. Cette étude a été réalisée en 2004, dans le cadre d'une recherche collaborative plus vaste, 
qui visait l'étude de l'implantation d'un programme préliminaire d'éveil aux langues (ÉLODiL) et des représentations des élèves de deux classes régulières de $5^{\text {e }}$ et $6^{\text {e }}$ années ainsi que de deux classes d'accueil ${ }^{10}$. La collecte de données de cette étude exploratoire, inspirée des approches ethnographiques, a donc eu lieu pendant la mise en ouvre des activités ÉLODiL.

Selon Harry Wolcott (1997), le mot ethnographie sous-tend la création d'une image d'une certaine «façon de vivre ". Cette création implique la compréhension de la logique de la population dans un lieu donné. Nos observations hebdomadaires (d'une durée de 90 minutes pendant 16 semaines) avaient pour objectif de comprendre la " logique linguistique " de nos neuf élèves ciblés, dans une classe pluriethnique à Montréal. Cette méthode nous a permis d'être au cœur même de l'action. Les interactions avec les élèves nous ont donné la possibilité de créer une relation privilégiée avec eux, sans qu'ils soient intimidés par notre présence. Les activités ÉLODiL et notre présence ont donc encouragé les élèves à s'exprimer sur le sujet des langues (ce qui, dans ce milieu, peut s'avérer délicat) dans un climat de confiance.

\section{Cueillette des donNées}

Parmi l'ensemble des résultats, cet article ciblera particulièrement les réponses des élèves portant sur la diversité linguistique, recueillies lors de groupes de discussion, d'une durée d'environ 40 à 60 minutes, et qui ont eu lieu à la fin du projet scolaire ÉLODiL, après quatre mois d'intervention. Ces entrevues ont été enregistrées et retranscrites. Même si ce n'était pas l'objet de la recherche et que nous n'avons pas cherché à le vérifier, il est possible que les activités ÉLODiL aient eu une certaine influence sur les réponses des élèves. Les élèves ont été séparés en deux groupes de discussion : celui des élèves de $5^{\mathrm{e}}$ année $(\mathrm{N}=4)$, et celui des élèves de $6^{\mathrm{e}}$ année $(\mathrm{N}=5)$. Un guide pour diriger ces groupes de discussion a été élaboré par les deux chercheures puisque, à leur connaissance, aucun guide de discussion pour des élèves de cet âge n'était disponible pour recueillir les représentations et les attitudes à l'égard des langues et de la diversité linguistique ${ }^{11}$. Parmi les sujets abordés, trois thèmes spécifiques seront étudiés dans le cadre de cet article. Pour le premier, l'appréciation des langues, nous avons fait l'analyse des réponses obtenues à la question suivante : " Estce que d'après vous, il y a des raisons qui font que vous trouvez une langue belle/pas belle? ». Pour les deux autres thèmes, soit le maintien de la langue maternelle et les langues et la diversité linguistique dans le monde, des mises en situation hypothétiques ont été proposées afin de recueillir les réponses, réactions et commentaires sur les questions de langue(s) maternelle(s) et/ ou d'origine et de langue universelle. Les deux mises en situation sont les suivantes : 
[Thème 2] Si vous pouviez choisir votre langue maternelle, laquelle choisiriezvous et pourquoi ?

[Thème 3] Si vous pouviez, d'un coup de baguette magique, faire qu'il n'y ait qu'une seule langue parlée sur la terre, pensez-vous que vous le feriez ? Selon vous, est-ce une bonne idée ou une mauvaise idée?

Afin de sélectionner notre échantillon, nous avons demandé à la titulaire d'identifier des élèves représentatifs de sa classe, dans ce milieu montréalais spécifique $^{12}$. Les données sociodémographiques et linguistiques de ces élèves se trouvent dans le tableau qui suit.

Notre échantillon est constitué de deux élèves monolingues (Elsa et Simon), de cinq élèves bilingues (Cyril, Daniel, Esteban, Kelly et Tuyet) et finalement de deux élèves plurilingues (Elijah et Kim). Nous inspirant de la définition de Grosjean (1984), nous définissons le bilingue ou le plurilingue comme étant une personne qui utilise quotidiennement deux ou plusieurs langues, écartant ainsi l'idée d'une nécessaire maîtrise parfaite de ces langues. Il est intéressant de noter que trois élèves (Simon, Kelly et Elsa) sont quotidiennement en contact avec une langue familiale qu'ils ne maîtrisent pas, comme le montre le tableau qui suit.

Lors des activités ÉLODiL, ces enfants étaient divisés en deux sousgroupes : celui des $5^{\mathrm{e}}$ année et celui des $6^{\text {e }}$ année. Une fois les données des entrevues transcrites, un premier niveau d'analyse a été entrepris, ce que
LeCompte et Preissle (1993) appellent scanning, soit une relecture de ces entrevues. Le programme informatique N6 (2002) a ensuite été utilisé pour construire une hiérarchie de codes (tree node) et repérer les thèmes émergents.

\section{RÉSULTATS}

La discussion exposée dans les prochaines sections s'appuie sur des extraits d'entrevues de groupe. Des analyses plus poussées sur les représentations des élèves à l'égard du français, de l'anglais et de leur langue d'origine sont présentées dans le mémoire de maîtrise de Maraillet (2005) ${ }^{13}$. La présente analyse est organisée autour des trois thèmes suivants, tels qu'ils ont émergé des données issues des groupes de discussion : l'appréciation des langues, la possibilité de changer de langue maternelle et la question de la diversité versus un monopole linguistique.

\section{Appréciation des langues du monde}

Il semblerait que tous les élèves ayant répondu à la question leur demandant de dire ce qu'ils aimaient ou non dans une langue soient surtout séduits par les sons et les tonalités. En effet, Cyril, Tuyet, Elijah, Simon et Kelly affirment qu'ils aiment « l'accent " ou « la prononciation » de l'espagnol et de l'arabe. Par ailleurs, les raisons évoquées pour ne pas aimer une langue sont diverses. Le groupe des plus jeunes évoque le débit de parole. Ils semblent tous d'accord sur le fait que les locuteurs 


\begin{tabular}{|c|c|c|c|c|c|}
\hline & $\begin{array}{l}\text { NOM } \\
\text { (modifié pour des } \\
\text { questions de } \\
\text { confidentialité) } \\
\end{array}$ & $\begin{array}{l}\text { PAYS DE } \\
\text { NAISSANCE }\end{array}$ & $\begin{array}{l}\text { PAYS D'ORIGINE } \\
\text { DES PARENTS }\end{array}$ & $\begin{array}{l}\text { ARRIVÉE AU } \\
\text { QUÉBEC ET ÂGE } \\
\text { À L'ARRIVÉE }\end{array}$ & $\begin{array}{l}\text { CLASSE } \\
\text { D'ACCUEIL }\end{array}$ \\
\hline \multirow[t]{5}{*}{$6^{\mathrm{e}}$ année } & Simon (garçon) & Canada & $\begin{array}{l}\text { Cambodge/ } \\
\text { Québec }\end{array}$ & - & - \\
\hline & Daniel (garçon) & Canada & Cambodge & - & 1998-1999 \\
\hline & Tuyet (fille) & Belgique & Vietnam & 1999 à 7 ans & - \\
\hline & Elijah (garçon) & Philippines & Philippines & 1999 à 7 ans & $\begin{array}{l}1999-2000 \\
2000-2001\end{array}$ \\
\hline & Kelly (fille) & Philippines & Philippines & 1998 à 6 ans & 1998-1999 \\
\hline \multirow[t]{4}{*}{$5^{\mathrm{e}}$ année } & Kim (fille) & Philippines & Philippines & 2001 à 9 ans & $\begin{array}{l}2001-2002 \\
2002-2003 \\
\end{array}$ \\
\hline & Esteban (garçon) & Bolivie & Bolivie & 1994 à 2 ans & - \\
\hline & Elsa (fille) & Bénin & Bénin & 1998 à 5 ans & - \\
\hline & Cyril (garçon) & Canada & Zaïre/Jamaïque & - & - \\
\hline
\end{tabular}

\begin{tabular}{|c|c|c|c|c|}
\hline & NOM & $\begin{array}{l}\text { PAYS D'ORIGINE } \\
\text { DES PARENTS }\end{array}$ & $\begin{array}{l}\text { LANGUES } \\
\text { MAÎTRISÉES }\end{array}$ & $\begin{array}{l}\text { LANGUES PARLÉES DANS } \\
\text { LA FAMILLE }\end{array}$ \\
\hline \multirow[t]{5}{*}{$6^{\mathrm{e}}$ année } & Simon (garçon) & $\begin{array}{l}\text { Cambodge/ } \\
\text { Québec }\end{array}$ & Français & Français, khmer \\
\hline & Daniel* (garçon) & Cambodge & Français, khmer & Khmer \\
\hline & Tuyet (fille) & Vietnam & Français, vietnamien & Français, vietnamien \\
\hline & Elijah* (garçon) & Philippines & $\begin{array}{l}\text { Anglais, tagalog, } \\
\text { bisaya }\end{array}$ & Anglais, tagalog \\
\hline & Kelly* (fille) & Philippines & Anglais & Anglais, tagalog \\
\hline \multirow[t]{4}{*}{$5^{\mathrm{e}}$ année } & Kim*(fille) & Philippines & Anglais, tagalog & Anglais, tagalog \\
\hline & Esteban (garçon) & Bolivie & Français, espagnol & Espagnol \\
\hline & Elsa (fille) & Bénin & Français & Français, fon \\
\hline & Cyril (garçon) & Zaïre/Jamaïque & Anglais & Anglais \\
\hline
\end{tabular}

(L'étoile [*] rappelle que l'enfant a fréquenté une classe d'accueil; les langues soulignées dans la dernière colonne sont parlées dans la famille, mais ne sont pas maîtrisées par les sujets [d'après les informations fournies par les enfants]).

des langues indiennes parlent « trop vite ", et qu'il s'agit là d'un facteur négatif. Notons que ce groupe ne comprend aucun locuteur d'une de ces langues. Toutefois, plus tard dans la conversation, cette affirmation est nuancée par l'ensemble du groupe qui souligne que pour une personne qui ne comprend pas la langue, le débit peut sembler trop rapide. Ils citent l'espagnol, dont le débit rapide est considéré comme amusant. Pour le groupe des plus vieux, 
la difficulté de l'apprentissage est évoquée par Elijah. En effet, il affirme être attiré par l'espagnol, car cette langue ressemble au tagalog qu'il maîtrise déjà, et qu'elle sera donc, dans son cas, facile à apprendre. Par contre, il dit ne pas aimer « les langues qui sont difficiles à apprendre ". Simon, quant à lui, affirme qu'il n'aime pas «le pays, le style de vie, comme l'Afghanistan et l'Inde " et que cela influence son appréciation de leurs langues. Signalons que nos observations et nos conversations avec Simon révèlent un contact très fréquent avec la télévision. On peut donc se demander quel rôle ont pu jouer les nouvelles qui y sont présentées dans la formation de ses représentations. Finalement, Daniel est particulièrement silencieux pendant que ses camarades s'expliquent et répond qu'il ne sait pas à chaque fois qu'il est interrogé. De façon tout aussi prudente, Elijah, et plus tard Tuyet, font valoir la part subjective de leurs réponses en mentionnant que «ça dépend des personnes ».

En interrogeant les élèves sur les langues en général, nous avons donc pu noter plusieurs raisons affectives et sociales qui les pousseraient ou non à apprendre des langues et qui, selon Gardner (1985), seraient susceptibles de les influencer dans la réussite de ces apprentissages. Une grande partie de nos neuf élèves semble attirée par les sonorités hispaniques. On peut se demander si c'est parce que les élèves sont en contact avec un grand nombre de locuteurs de cette langue, ou bien parce que les médias et leur entourage leur disent que ce serait la troisième langue la plus parlée au monde (groupe de discussion, $5^{\circ}$ année), ou encore parce qu'elle présente des similarités avec le français et le tagalog (qui, rappelons-le, est parlé par un grand nombre d'élèves de cette école). Par contre, les langues indiennes, pourtant également assez présentes dans l'école, n'ont pas une cote aussi élevée. Comme nous l'indiquions, le débit rapide de ces langues est perçu négativement, alors que le débit rapide de l'espagnol est considéré comme amusant. Nous pouvons supposer que certains mots espagnols, d'origine latine, sont reconnus par les élèves et que, par conséquent, ils s'en sentent plus proches.

Notons aussi que la perception de la facilité d'apprentissage d'une langue, qu'elle soit due ou non à sa ressemblance avec la langue maternelle, semble aussi être un facteur de motivation. À l'inverse, si une langue est perçue comme trop difficile, certains élèves évoquent cette raison pour ne pas vouloir l'apprendre. Nous pouvons nous demander d'où proviennent ces représentations quant au niveau de difficulté des langues et quel impact ces représentations ont sur leur éventuel apprentissage. Finalement, nos données révèlent un point qui n’a pas été soulevé par les recherches antérieures : bien que notre étude ne nous permette pas de généraliser, nous pouvons proposer l'hypothèse selon laquelle les médias jouent un rôle non négligeable dans la motivation à apprendre ou non une langue. 
Sur la possibilité de changer de langue maternelle

Lorsque nous avons demandé aux élèves de nos deux groupes s'ils aimeraient changer de langue maternelle, la majorité d'entre eux, soit cinq élèves sur neuf, ont choisi de maintenir leur langue d'origine. Les élèves ayant fait ce choix ont comme langue maternelle le tagalog (Elijah et Kim), l'espagnol (Esteban), l'anglais (Kelly) et le vietnamien (Tuyet). La raison principale semble être l'attachement sentimental et culturel à cette langue, comme l'exprime Tuyet :

«Moi je garderais le vietnamien [comme langue maternelle], parce que si je ne le savais pas je ne comprendrais pas la culture de mon pays et je suis fière de mon pays. "

Il est toutefois intéressant de constater que Kelly a choisi le tagalog, langue utilisée par ses parents à la maison mais qu'elle ne maîtrise pas. Par ailleurs, trois élèves sur neuf ont choisi ce qu'on appelle des "grandes langues", l'anglais (Daniel et Simon) et l'espagnol (Elsa). La raison principale de ce choix semble être le besoin de connaître une langue qui est largement utilisée autour d'eux et qu'ils ne maîtrisent pas : " [je choisis l'] Anglais !!! [comme langue maternelle] Parce que c'est utile, et moi je ne la parle pas. » (Simon). Finalement, le dernier élève, Cyril, a choisi avec beaucoup d'enthousiasme le japonais. Il explique ce choix quelque peu surprenant par un besoin de connaître une langue un peu plus exotique que celles qu'il connaît déjà, à savoir le français et l'anglais. En effet, on peut penser que dans un contexte multiethnique, Cyril se sent un peu trop « ordinaire».

Rappelons que l'étude de Beauchesne et Hensler (1987) avait révélé que la majorité des enseignants qu'ils avaient interrogés à l'époque pensaient que leurs élèves d'origine immigrante souhaitaient maintenir leur langue d'origine et que ces enseignants étaient partagés quant à l'impact positif ou négatif de ce maintien. Vingt ans après, notre étude permet de redonner la parole à un petit nombre de ces jeunes élèves. Nos résultats indiquent que, pour la majorité des enfants, le maintien de la langue d'origine est perçu comme positif, voire nécessaire, et que, pour les autres, il est dépassé par le besoin de connaître une autre langue, considérée comme plus utile, comme l'anglais ou l'espagnol, ou plus exotique, comme le japonais. Cependant, nous n'avons relevé aucun commentaire selon lequel maintenir sa langue d'origine aurait un impact négatif. Nous en discuterons dans la section suivante, mais nous pouvons d'ores et déjà faire un lien avec le modèle gravitationnel de Calvet, basé sur les différentes fonctions des langues.

\section{Diversité versus monopole linguistique}

Lorsque nous avons demandé aux élèves s'ils pensaient que le fait de 
n'avoir qu'une seule langue parlée sur la terre était une bonne idée ou non, ils ont répondu de façon similaire dans les deux groupes. Sans l'ombre d'un doute, et sans prendre le temps de réfléchir, les élèves les plus jeunes, ceux de $5^{\mathrm{e}}$ année, sont unanimes : ce n'est pas une bonne idée. Elsa est la première à donner une justification, qui se trouve être le rejet de l'uniformité : "Ça va être comme trop plate, on va toujours parler la même chose!". Cette remarque nous laisse perplexes puisque Elsa ne parle qu'une seule langue. Alors qu'elle n'a fait aucun commentaire sur le fait qu'elle trouvait que sa situation était ennuyante, elle semble être sensibilisée au fait que la diversité linguistique rend la vie plus intéressante, ce que quatre mois d'éveil aux langues ont pu favoriser. Puis Esteban, d'un point de vue très personnel, réalise que la diversité linguistique est bien utile puisqu'elle implique que certaines personnes ne peuvent pas se comprendre, ce qui, à l'inverse, crée une complicité entre ceux qui partagent la même langue :

«Parce que on pourra pas parler dans notre langue pour que quelqu'un ne comprenne pas, et puis on va tous avoir la même écriture là, tout va être pareil, ça va être comme plate et poche.» (Esteban).

Esteban élargit les conséquences à l'uniformité des écritures, dont il semble apprécier la diversité, et finit par reprendre l'argument d'Elsa sur l'ennui que serait un monde « monolingue ». Les pensées de Cyril sont davantage orientées sur son rapport avec le monde. En effet, il pense à l'expérience intéressante de voyager dans des pays inconnus et d'apprendre les langues qui y sont parlées.

Comme les $5^{\mathrm{e}}$ année, les élèves de $6^{\mathrm{e}}$ année répondent tous négativement à la question. La première justification est mise de l'avant par Tuyet qui fait un rapprochement entre les langues et les cultures : "Parce que s'il y a une seule langue, il y a une seule culture, et moi je ne veux pas cela ". Simon complète l'argument de Tuyet, selon une perspective davantage anthropologique, en soulignant l'importance de la notion de découverte :

\begin{abstract}
"Moi non, parce qu'on va penser que c'est pareil partout dans le monde et on ne pourra plus découvrir les autres cultures. On ne va plus rien découvrir. "(Simon).
\end{abstract}

La chercheure décide de relancer la question, pour vérifier si certains élèves auraient changé d'avis. Mais non : Elijah annonce que sa propre diversité linguistique, son plurilinguisme, lui procure beaucoup de fierté. Daniel, qui ne semble pas avoir d'autres arguments, reprend celui des cultures. Quant à Kelly, de façon similaire à Elijah et à Esteban, elle souligne l'unicité des individus et explique que celle-ci serait perdue si nous parlions tous la même langue dans le monde. 
Alors que l'étude de McAndrew et al. (1999) a montré que le taux de présence de la langue française dans les dix écoles primaires observées est très élevé et que celui de l'anglais et des langues d'origine est faible, notre recherche souligne que ces usages n'impliquent pas forcément le rejet de la diversité linguistique et que, bien au contraire, il est tout à fait possible que les élèves des écoles multiethniques la valorisent grandement. Par ailleurs, comme nous l'avons mentionné brièvement dans la première partie de nos résultats, les réponses de nos sujets se rapprochent de la vision du modèle gravitationnel de Calvet (2002). En effet, il semblerait qu'ils voient dans leur langue d'origine un lien essentiel avec leur famille, une caractéristique identitaire qui les rend uniques et une source de fierté. Les autres langues choisies et identifiées semblent remplir une fonction différente dans leur vie, comme être capable de communiquer avec le reste du monde en anglais, ou encore maîtriser le japonais pour connaître une langue inconnue de tous. Cependant, on distingue aussi dans cette vision un sentiment profond que le monde est plus intéressant si ses langues sont diversifiées, comme le revendique Hagège (2002).

\section{Conclusion}

Comme pour toutes les études portant sur un nombre restreint de sujets, nous ne pouvons généraliser nos résultats à l'ensemble de la clientèle pluriethnique des écoles montréalaises. D'autres études devront donc recueillir des données dans des milieux semblables, et en explorer d'autres, plus homogènes. Par ailleurs, il convient de préciser que le contexte de cette recherche (le quartier, le projet ÉLODiL) était volontairement chargé d'enjeux linguistiques, ce qui a peut-être généré des réponses perçues comme socialement désirables. Cependant, certains extraits confirment que cela n'a pas toujours été le cas. Cette dynamique linguistique intéressante a, par ailleurs, fait émerger plusieurs points qui nous apparaissent particulièrement pertinents.

Premièrement, les réponses des élèves révèlent les représentations qu'ils ont de leurs besoins. Si la majorité de nos répondants se disent satisfaits de leur situation linguistique, plusieurs enfants qui semblent avoir constaté leur "faiblesse linguistique" saisissent l'occasion pour imaginer leur vie autrement, que ce soit pour manifester leur appartenance identitaire, pour combler ce qui est perçu comme une lacune linguistique, ou encore pour se rendre "linguistiquement" plus intéressant. Par ailleurs, nos données sur l'appréciation des élèves à l'égard des langues demeurent limitées : seuls quelques éléments phonologiques sont ressortis de nos discussions. Ce constat nous amène à nous demander si nos répondants ne se sont pas autocensurés, que ce soit par politesse ou conscience que certains propos n'étaient pas «politiquement corrects ». De plus, on peut s'interroger sur l'impact des médias quant aux représentations à l'égard des langues et de leurs locuteurs. Finalement, 
les réponses recueillies illustrent ce que nous pouvons appeler la compétence sociolinguistique des élèves. On retrouve tout à la fois l'idée de reconnaissance et de valorisation d'une langue donnée, permettant aux hommes et aux femmes de la terre de communiquer, et celle de reconnaissance et de valorisation de la grande diversité des langues dans le monde, reflétant la variété des personnes qui s'y trouvent. Selon nous, cette position témoigne d'une grande maturité chez ces enfants évoluant dans une société où la nature des relations interlinguistiques est ambiguë et tendue. Pouvons-nous dire que les enfants de cette étude ont bénéficié d'un environnement qui les a encouragés à réfléchir sur la question des langues ? C'est fort probable. En ce qui concerne la protection des langues, dans la mesure où ils y verront le reflet et la fierté d'une communauté qu'il faut protéger, on peut penser que ces enfants seront prêts à s’engager dans le débat.

\section{Notes}

${ }^{1}$ Nous remercions les organismes subventionnaires suivants qui nous ont permis de réaliser cette recherche : Centre de recherche Immigration et métropoles (projet Métropolis), Conseil de recherche en sciences humaines du Canada/ Patrimoine Canadien.

${ }^{2}$ Ces commissions ont été séparées sur la base confessionnelle jusqu'en 1998. À cette date, elles sont devenues linguistiques (réseaux francophone et anglophone).

${ }^{3} \mathrm{Au}$ Québec, le terme allophone désigne une personne dont la langue maternelle n'est ni le français ni l'anglais.

${ }^{4}$ En 1990, ces programmes connaissent une expansion limitée et touchent quelques 7000 élèves pour environ quatorze langues (Laurier et Témisjian 2003, cité dans Armand 2005).

${ }^{5}$ Cette étude a été réalisée au moyen d'entrevues et de questionnaires auprès de 621 répondants (enseignants, professionnels des services aux élèves, administrateurs, parents et élèves québécois et d'immigration récente) présents dans 64 écoles primaires et 12 écoles secondaires de langue française, de 5 des 8 commissions scolaires de l'île de Montréal.

${ }^{6}$ Recherche subventionnée par le Conseil de recherche en sciences humaines du Canada/ Patrimoine Canada - Les questions de multiculturalisme : F. Armand et D. Dagenais (2004-2005). Conception et évaluation d'un programme d'éveil au langage et d'ouverture à la diversité linguistique; et par le projet Métropolis à Montréal et à Vancouver.

${ }^{7}$ Recherche subventionnée par le Conseil de recherche en sciences humaines du Canada : D. Dagenais, F. Armand, P. Lamarre, D. Moore et C. Sabatier (2005-2008). Éveil aux langues et à la diversité linguistique dans deux métropoles canadiennes.

${ }^{8}$ Parmi ces facteurs, on retrouve des situations de contacts plus ou moins formelles, les antécédents linguistiques des élèves en présence, ainsi que tout autre facteur institutionnel qui aurait pu s'avérer pertinent.

${ }^{9}$ Le $3^{\mathrm{e}}$ cycle correspond, dans le système scolaire québécois, à la $5^{\mathrm{e}}$ et à la $6^{\mathrm{e}}$ année du primaire. ${ }^{10}$ Ces classes d'accueil à effectif réduit et d'une durée prévue généralement de dix mois, ont pour objectif principal de faire acquérir à l'élève nouvellement arrivé au Québec des habiletés langagières minimales correspondant à son âge, à ses besoins et à ses intérêts, avant d'intégrer une classe régulière (Armand 2005).

${ }^{11}$ En effet, dans l'étude d'Evlang, les entretiens avec les élèves ont servi à recueillir des données pour : 1- améliorer les supports, 2- évaluer comment les élèves s'étaient approprié la démarche Evlang, 3- confronter le comportement et la déclaration des élèves en classe et 4- Analyser les représentations qu'avaient les élèves du travail Evlang et du sens qu'ils ont donné à leur apprentissage (Candelier 2003). Pour les représentations et les attitudes des élèves à l'égard des langues, les chercheurs Evlang ont préféré utiliser des tâches à réaliser par les élèves. Le seul exemple publié consistait à remplir une bande dessinée. Celle-ci montrait deux enfants qui jouent ensemble. Un troisième enfant arrive et commence à parler dans une autre langue. L'élève avait alors deux options pour terminer la bande dessinée : il choisissait soit l'image dans laquelle les deux enfants qui parlent la même langue s'en vont, soit celle qui montre les trois enfants qui jouent ensemble. L'élève devait aussi remplir les bulles vierges de la dernière vignette choisie et avait ainsi la possibilité d'écrire dans une autre langue. Cette tâche permettait donc de recueillir des données sur l'attitude des élèves 
à l'égard de leur langue d'origine et des situations exolingues, mais pas forcément sur leurs représentations à l'égard de la diversité linguistique en général.

12 On peut considérer que cet échantillon est effectivement représentatif pour plusieurs raisons : par exemple, on retrouve un équilibre d'élèves issus de l'immigration de première et de deuxième générations, d'élèves qui ont fréquenté ou non une classe d'accueil, de filles et de garçons, ou encore une présence marquée d'élèves originaires des Philippines, d'où sont issus un grand nombre d'immigrants récemment installés dans le quartier entourant l'école.

${ }^{13}$ Mémoire réalisé sous la direction de Françoise Armand, et sous la co-direction de Patricia Lamarre, professeures au département de didactique, Université de Montréal.

\section{Bibliographie}

Allport, G., 1935. "Attitude ", in C.M. Murchison (ed.), Handbook of social psychology. Worcester, Mass, Clark University Press, p. 798-884.

Armand, F., 2005. "Les élèves immigrants nouvellement arrivés et l'école québécoise ", Revue Santé, société et solidarité, $\mathrm{n}^{\circ}$ 1, p. 141-152.

Armand, F. et D. Dagenais, 2005. « Langues en contexte d'immigration : éveiller au langage et à la diversité linguistique en milieu scolaire ", Revue de l'Association des études canadiennes, numéro spécial printemps, p. 110-113.

Beauchesne, A. et H. Hensler, 1987. L'école française à clientèle pluriethnique de l'île de Montréal : situation du français et intégration psychosociale des élèves. Montréal, Conseil de la langue française, Coll. Document 25, 618 p.

Calvet, L.-J., 2002. Le marché aux langues. Les effets linguistiques de la mondialisation. Paris, Éditions Plon, 220 p.

Candelier, M., 2003. L'éveil aux langues à l'école primaire. Evlang : bilan d'une innovation européenne. Bruxelles, De Boeck \& Larcier, 379 p.

Chancy, M., 1985. L'École québécoise et les communautés culturelles. Ministère de
l'Éducation du Québec, 180 p.

Comité de gestion de la taxe scolaire de l'île de Montréal, 2003. Portrait socioculturel des élèves inscrits dans les écoles publiques de l'île de Montréal. Inscription au 30 septembre 2002. Montréal, 417 p.

Gardner, R. C., 1985. Social Psychology and Second Language Learning. The Role of Attitudes and Motivation. London, Edward Arnold Publishers, 208 p.

Garrett, P. et C. James, 1992. « Language awareness : a way ahead ", in C. James et P. Garett (ed.), Language awareness in the classroom. London, Longman, p. 306318.

Grosjean, F., 1984. " Le bilinguisme: vivre avec deux langues ", Travaux Neuchâtelois de Linguistique (Tranel), $\mathrm{n}^{\mathrm{o}}$ 7, p. 15-42.

Hagège, C., 2002. Halte à la mort des langues. Paris, Éditions Odile Jacob, 402 p.

Hawkins, E., 1987. Awareness of Language: An introduction. Cambridge, Cambridge University Press, 226 p.

Jodelet, D., 1984. « Représentation sociale : phénomène, concept et théorie ", in S. Moscovici (éd.), Psychologie sociale. Paris, Presses Universitaires de France, p. 357-378.

Laurier, M. et K. Témisjian, 2003. «Représentations des acteurs intervenant dans les programmes d'enseignement des langues d'origine au Québec ", Communication présentée à la $8^{e}$ Conférence internationale Metropolis. Vienne, 16 septembre 2003.

LeCompte, M. et J. Preissle, 1993. Ethnography and Qualitative Design in Educational Research. Second Edition, San Diego, CA, Academic Press Inc., 292 p.

Locher, U., 1993. Les jeunes et la langue. Tome 1. Usages et attitudes linguistiques des jeunes qui étudient en français (de la $4^{e}$ année $d u$ secondaire à la fin $d u$ collégial). Dossier 39. Québec, Les Publications du Québec, 204 p.

Locher, U., 1994. Les jeunes et la langue. Tome 2. Usages et attitudes linguistiques 
des jeunes qui étudient en anglais (de la $4^{e}$ année $d u$ secondaire à la fin $d u$ collégial). Dossier 40. Québec, Les Publications du Québec, 196 p.

Maraillet, É., 2005. Étude des représentations linguistiques d'élèves au $3^{e}$ cycle $d u$ primaire, en milieu pluriethnique à Montréal, lors d'un projet d'éveil aux langues. Mémoire de Maîtrise, Département de didactique, Faculté des Sciences de l'éducation, Université de Montréal, 262 p. Disponible en ligne : http://im.metropolis.net/research-policy/ research content/doc/memoireMaraillet.pdf.

Masgoret, A.-M. et R.C. Gardner, 2003. "Attitudes, Motivation, and Second Language Learning: A MetaAnalysis of studies conducted by Gardner and Associates ", Language Learning, vol. 53, $\mathrm{n}^{\circ}$ 1, p. 123-163.

McAndrew, M. et P. Lamarre, 1996. «The integration of Ethnic Minority Students Fifteen Years after Bill 101 : Linguistic and Cultural Issues Confronting Quebec's French Language Schools ", Canadian Ethnic Studies, vol. XXVIII, $\mathrm{n}^{\circ} 2$, p. 4063.

McAndrew, M. et J.-P. Proulx, 2000. « Éducation et ethnicité au Québec : un portrait d'ensemble ", in M. McAndrew et F. Gagnon (dir.), Relations ethniques et éducation dans les sociétés divisées (Québec, Irlande du Nord, Catalogne et Belgique). Paris, Montréal, L'Harmattan, p. 85-110.

McAndrew, M., C. Veltman, F. Lemire et J. Rossell, 1999. Concentration ethnique et usages linguistiques en milieu scolaire. Montréal, Immigration et Métropoles, 171 p.

Ministère de l'Éducation du Québec (MEQ), 1998. Une école d'avenir. Politique d'intégration scolaire et d'éducation interculturelle. Québec, 48 p.

Ministère de l'Éducation du Québec (MEQ), 2001. Programme de formation de l'École québécoise : éducation préscolaire et enseignement primaire. Québec, $350 \mathrm{p}$.

Ministère des Relations avec les citoyens et de l'Immigration (MRCI), 2003. Caractéristiques de l'immigration au
Québec- Statistiques. Québec, Bibliothèque nationale du Québec, 39 p.

Moscovici, S., 1984. Psychologie sociale. Paris, Presses Universitaires de France, 596 p.

N6, 2002. Non-numerical Unstructed Data Indexing Searching \& Theorizing. Qualitative data analysis program. Version 6.0 Melbourne, Autralia, QSR International Pty Ltd.

Sénéchal, G., 1987. Les allophones et les anglophones inscrits à l'école française. Sondage sur les attitudes et les comportements linguistiques. Québec, Conseil de la langue française, $208 \mathrm{p}$.

Singy, P., 1997. " Le rôle de l'école dans l'élaboration des représentations linguistiques ", in M. Matthey (éd.), Les langues et leurs images. Neuchâtel, IRDP/ Éditeur, p. 277-283.

Stefanescu, A. et P. Georgeault (dir.), 2005. Le Français au Québec, les nouveaux défis. Québec, Les Éditions Fides (Saint Laurent), en partenariat avec le Conseil supérieur de la langue française, $622 \mathrm{p}$.

Wolcott, H., 1997. « Ethnography Research in Education ", in R. Jaeger (ed.), Complementary methods: For research in education ( $2^{\text {nd }} \mathrm{ed}$.). Washington, DC, AERA, p. 425-446. 